\title{
Studiendesigns mit unvollständiger Aufklärung der Versuchspersonen
}

\author{
Der Entwurf des Humanforschungsgesetzes sieht Ausnahmen von der Verpflichtung \\ zur vollständigen Aufklärung einer Versuchsperson vor. Dies birgt erhebliche ethi- \\ sche Probleme, vor allem wenn es hauptsächlich dazu dient, die Bereitschaft zur Stu- \\ dienteilnahme zu steigern.
}

\footnotetext{
Die Literaturangaben finden sich im Internet unter www.saez.ch $\rightarrow$ aktuelle Nummer oder $\rightarrow$ Archiv $\rightarrow$ $2010 \rightarrow 25$
}

Interessenverbindungen: keine

Korrespondenz:

Dr. med. Peter Kleist

GlaxoSmithKline AG

Talstrasse 3-5

CH-3053 Münchenbuchsee

Tel. 0318622460

peter.m.kleist@gsk.com

\section{Einführung}

Nach der Annahme des Verfassungsartikels über die Forschung am Menschen ist der Weg für das Humanforschungsgesetz geebnet. Der aktuelle, in die parlamentarische Beratung eingehende Gesetzesentwurf [1]* sieht mit Artikel 18 Ausnahmen von der Verpflichtung zur vollständigen Aufklärung einer Versuchsperson vor Beginn des Forschungsprojektes vor - wenn dies aus methodischen Gründen zwingend ist und wenn mit dem Forschungsprojekt nur minimale Risiken und Belastungen verbunden sind.

Zunächst stellt sich die Frage, ob eine unter ethischen Gesichtspunkten fragwürdige Ausnahmebestimmung auf Gesetzesstufe nicht einen unangemessen hohen Stellenwert erhält. Davon abgesehen ist der Artikel $18 \mathrm{zu}$ unpräzise. Was sind «zwingende methodische Gründe»? Gilt der Artikel generell für alle Forschungsbereiche? Ob einem Forschungsprojekt, das grundlegende Rechte von Versuchspersonen einschränkt, auch eine «zwingend zu beantwortende Frage» zugrunde liegen muss, wird leider gar nicht thematisiert.

In ihrem informativen und lesenswerten Artikel «Informed Consent - Balance zwischen Patientenschutz und Forschungsinteressen» [2] geben M. Fässler und N. Biller-Andorno einen Überblick über Studiendesigns mit Modifikationen des Informed ConsentProzesses. Der folgende Beitrag hinterfragt die beschriebenen Studientypen aus methodologischer und ethischer Sicht, wobei der Fokus auf Heilmittelstudien gerichtet wird.

\section{Grundvoraussetzungen}

Um als Alternative zum Standard, d.h. der randomisierten kontrollierten Studie, überhaupt in Betracht zu kommen, müssen aus meiner Sicht bei Studien mit unvollständiger Aufklärung der Versuchspersonen die folgenden fünf Voraussetzungen kumulativ erfüllt sein:

- Eine Verblindung ist nicht möglich, weil völlig unterschiedliche Massnahmen untersucht werden (z.B. konventionelle vs. operative Therapie, Screening vs. kein Screening).

\section{Information incomplète des sujets impliqués dans des essais cliniques}

L'actuel projet de loi relatif à la recherche sur l'être humain prévoit des exceptions quant à l'obligation d'informer de manière exhaustive les sujets. Les essais cliniques exemptés du devoir d'informer sont non seulement douteux d'un point de vue éthique, mais présentent également des lacunes sur le plan méthodologique. «Des raisons méthodologiques», telles que les préconise l'actuel projet de loi, ne devrait s'imposer que pour des cas rares. Une information incomplète dans le cadre d'une étude ne devrait pouvoir se justifier que si elle concerne des questions brûlantes de santé publique. Ce principe ne doit en aucun cas être toléré s'il ne vise qu'à faciliter le recrutement par les chercheurs de leurs sujets.

On touche là aux droits fondamentaux des sujets, il est donc nécessaire d'apporter de nettes précisions à la loi. Pour ce qui est des études sur les produits thérapeutiques en revanche, il n'y a pas lieu de prévoir une loi régissant les exceptions.

- Aus Sicht der Versuchspersonen sind die untersuchten Massnahmen nicht gleichwertig; z.B. wird einer attraktiven neuen Behandlung eindeutig der Vorzug vor der Standardbehandlung gegeben.

- Die Kenntnis der Alternativbehandlung führt mit sehr hoher Wahrscheinlichkeit zur sogenannten Kontamination; z. B. kann es bei den Versuchspersonen, die sich in der Kontrollgruppe wissen, zum Verlust der Compliance kommen oder die Kontroll- 
personen übernehmen die Massnahmen der Interventionsgruppe, wenn diese relativ leicht zugänglich sind (z. B. eine spezielle Diät).

- Es gibt definitiv keine andere Möglichkeit, die Forschungsfrage zu beantworten.

- Dem Forschungsprojekt selbst muss eine elementare Bedeutung für die Gesundheitsversorgung zukommen.

\section{Methodologische Probleme}

Der Ausdruck «zwingende methodische Gründe» erweckt den Anschein, dass Studiendesigns mit modifizierter Aufklärung «über jeden Zweifel erhaben sind». Nachfolgend sollen nur die wichtigsten methodologischen Probleme dieser Studientypen kurz skizziert werden.

\section{Zelen-Designs}

Den vom amerikanischen Statistiker Marvin Zelen vorgeschlagenen Studiendesigns liegt der Ansatz zugrunde, eine Versuchsperson erst nach Einschluss in die Studie, d.h. nach der Randomisierung über die ihr zugewiesene Therapie zu informieren und ihre Einwilligung einzuholen. Somit besteht die Möglichkeit, das Ziel des Projekts, die Tatsache der Randomisierung und das Vorhandensein einer Alternativtherapie zu verschweigen.

Eine sehr beschränkte praktische Bedeutung kommt gegenwärtig jedoch nur dem Double Consent Design von Zelen zu (siehe Abb. 1) [3]. Entgegen Zelens Einschätzung vor fast 30 Jahren, dass seine Studiendesigns zukünftig das Feld der randomisierten kontrollierten Studien dominieren werden [4], sind diese bis dato tatsächlich nur bei einer überschaubaren Anzahl publizierter Studien angewendet worden: Übersichtsarbeiten haben nur ca. 60 Studien zwischen

Abb. 1

Double Consent Design nach M. Zelen [3].

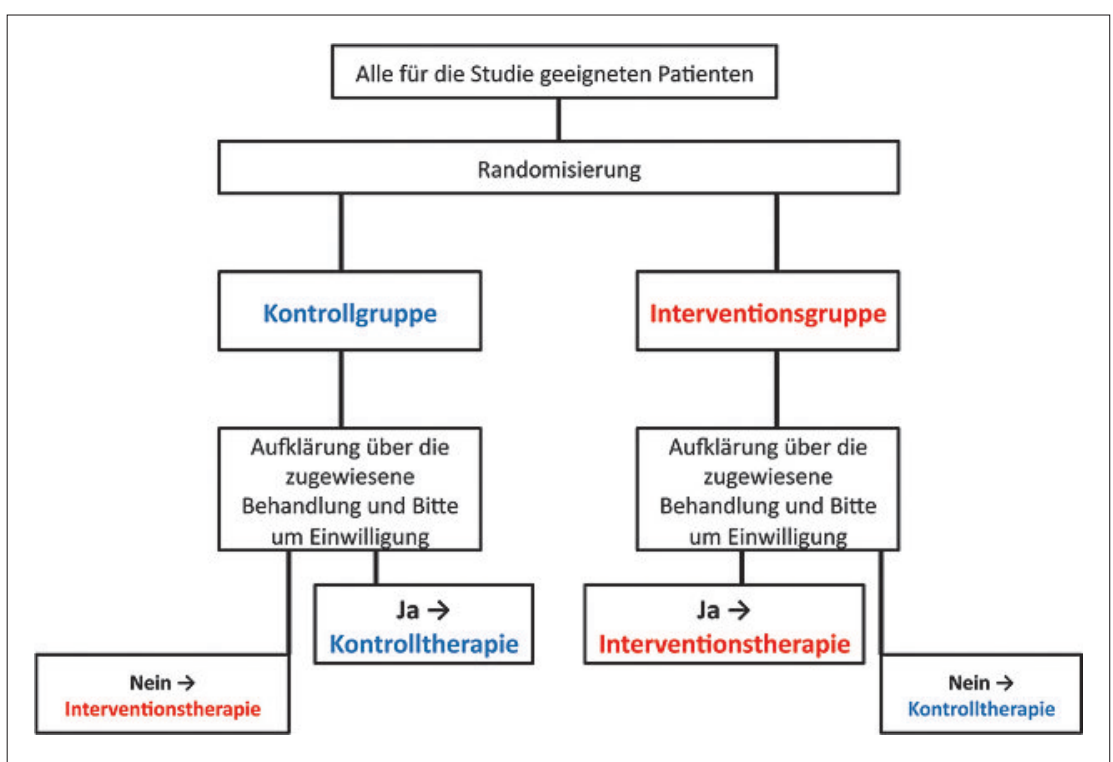

1979 und 2005 ausfindig machen können, weniger als die Hälfte davon waren Studien mit Heilmitteln, schwerpunktmässig im Bereich der Onkologie $[5,6]$.

Neben der ethischen Problematik sind die Gründe dafür wesentlich auch im methodologischen Bereich zu suchen. Lehnen Patienten in einer Studie mit Zelens Double Consent Design die ihnen zugewiesene Behandlung ab, erhalten sie die alternative Therapie. Da jedoch die Auswertung der Studie nach dem Intentionto-treat-Prinzip erfolgt (d.h. so wie randomisiert und nicht wie tatsächlich behandelt), unterliegt die beobachtete Differenz zwischen den untersuchten Behandlungen einem «Verdünnungseffekt». Bei niedriger Ablehnungsrate ist dieser Effekt zu vernachlässigen; überschreitet die Ablehnungsrate jedoch ein bestimmtes Ausmass, ist die Effektabschätzung einem starken Bias unterworfen. Ein Beispiel soll das verdeutlichen. Angenommen, die wahren Überlebensraten unter Behandlung A und B betragen 20\% bzw. 40\%. Wenn aber jeweils ein Fünftel der Patienten die ihnen zugewiesene Behandlung ablehnt und stattdessen die andere Behandlung erhält, betragen die beobachteten Überlebensraten $24 \%$ bzw. $36 \%$, und die wahre Behandlungsdifferenz von $20 \%$ schrumpft auf beobachtete $12 \%$ [7]. Um den Verdünnungseffekt zu kompensieren, ist die Stichprobengrösse zu erhöhen. Allerdings führt eine Ablehnungsrate von 15\% im Vergleich zu einer konventionellen randomisierten Studie bereits zu einer Verdopplung der benötigten Patientenanzahl.

Ist dies reine Theorie? Nein! Untersuchungen an Chemotherapiestudien mit Zelen-Design haben ergeben, dass die durchschnittliche Ablehnungs- resp. Transferrate zwischen 10\% und 36\%, im Mittel bei etwa $18 \%$ liegt [8]. Aufgrund der hohen Ablehnungsquote wechselten die Prüfer während laufender Studien in einigen Fällen sogar zum konventionellen Studiendesign über (Beispiel in [9]). Auch lässt sich der hypothetische Vorteil des Zelen-Designs, nämlich die Verhinderung einer «Kontamination», in der Praxis oft nicht zeigen. In der Mehrzahl der Chemotherapiestudien mit Zelen-Design waren die Noncompliance und die Ausfallrate in der Experimentalgruppe höher als in der Kontrollgruppe [6].

\section{Cluster-Randomisierungsstudien}

In Cluster-Randomisierungsstudien werden nicht individuelle Personen, sondern Personengruppen (Cluster) randomisiert. Cluster können z. B. Arztpraxen, Spitäler oder Pflegeheime, ggf. sogar ganze geographische Regionen sein. Da allen Patienten eines Clusters jeweils nur eine Behandlung angeboten wird, ist der Aufklärungs- und Einwilligungsprozess mit dem von Zelen-Design-Studien vergleichbar. Cluster-Randomisierungsstudien haben vor allem im Public HealthBereich einen gewissen Stellenwert. Ausser für die Effektivitätsbewertung von Impfprogrammen - hier spielt nicht nur der individuelle Impferfolg, sondern auch die Herdimmunität einer Population eine Rolle - kommt Cluster-Studien für reine Heilmittel- 
studien praktisch keine Bedeutung zu. Aufgrund der inhärenten methodologischen Probleme soll dennoch kurz auf dieses Studiendesign eingegangen werden.

Anders als bei herkömmlichen randomisierten Studien können die einzelnen Personen in einem Cluster nicht als unabhängige Grösse betrachtet werden; Personen in einem Cluster teilen mit hoher Wahrscheinlichkeit bestimmte Eigenschaften und sind sich daher ähnlicher als Personen aus verschiedenen Clustern [10]. Daraus resultiert unter Umständen eine eingeschränkte Vergleichbarkeit der Untersuchungsgruppen. Ein Beispiel: Am Ende einer Cluster-Randomisierungsstudie zum Brustkrebs-Screening bestand ein Ungleichgewicht zwischen beiden Studienarmen bezüglich des sozioökonomischen Status der Teilnehmerinnen, was die Auswertung und Interpretation der Ergebnisse erschwerte - trotz Einschluss von 87 Clustern und 50000 Frauen [11].

\section{Erhält eine unter ethischen Gesichtspunkten fragwürdige Ausnahmebestimmung auf Gesetzesstufe nicht einen unangemessen hohen Stellenwert?}

Aufgrund der fehlenden Verblindung einer Cluster-Randomisierungsstudie besteht die zweite Problematik in einem möglichen Selektionsbias, der auf zwei Ebenen auftreten kann: auf Clusterebene, wenn z. B. die weniger motivierten Cluster vorzugsweise der Kontrollgruppe zugeordnet werden; und auf Ebene der Versuchspersonen, wenn nicht alle Personen, sondern

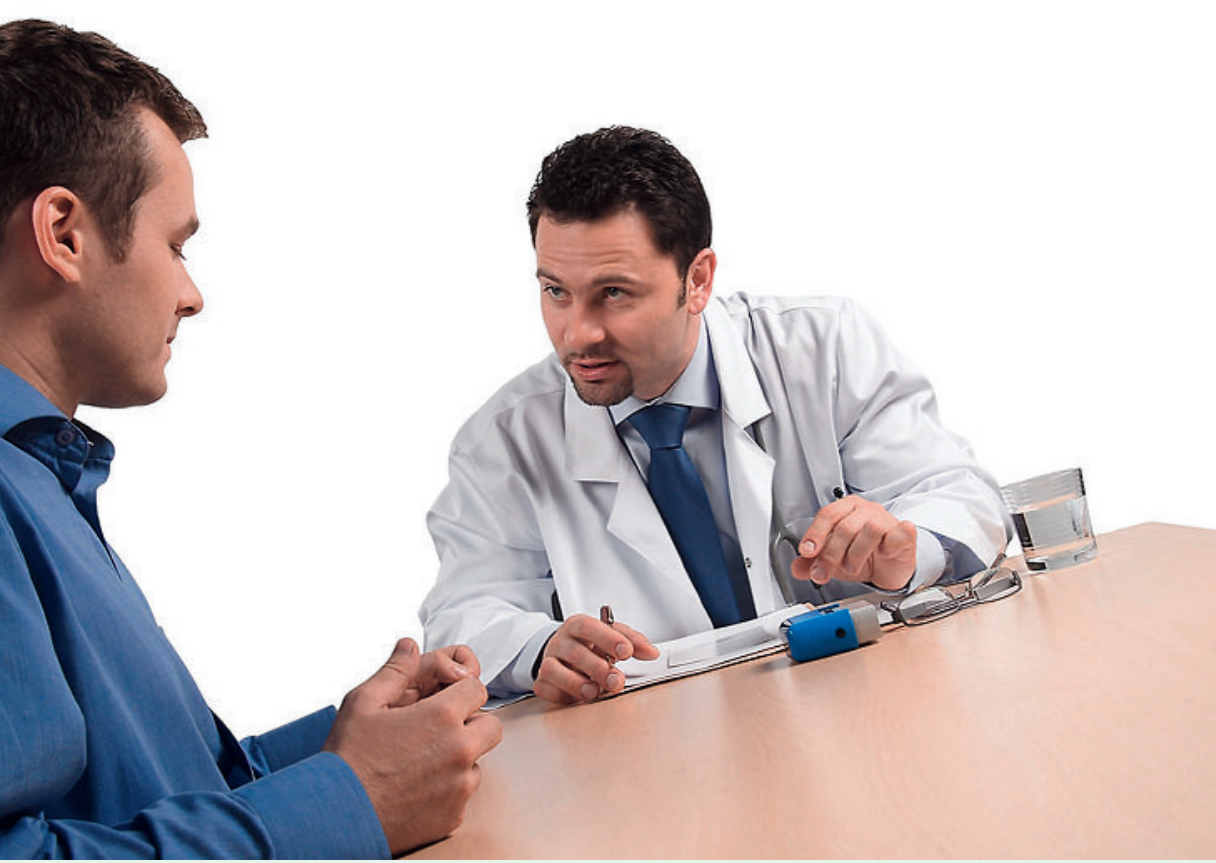

Vor Einschluss in eine Studie sollte die vollständige Aufklärung über deren Zweck, Ablauf, Aufwand, Belastung und Risiko immer gewährleistet sein. nur eine Auswahl von ihnen in die Studie einbezogen wird - dann nämlich werden Faktoren wie die Attraktivität einer Massnahme oder der durch sie erzeugte Aufwand mitentscheidend für die Teilnahme. Beide Formen des Selektionsbias können potentiell das Ergebnis einer Studie beeinflussen, weil die Personen in den Studienarmen unter Umständen nicht mehr vergleichbar sind, beispielsweise aufgrund Unterschiede im Schweregrad einer Erkrankung oder im Ausmass des gesundheitsbewussten Verhaltens [12]. So war z. B. in einer Studie zur Gesundheitsberatung von Personen mit erhöhtem Herzkreislaufrisiko die Prävalenz von Rauchern im Interventionsarm deutlich geringer; die Personen im Interventionsarm wiesen also per se ein geringeres Risiko für ischämische Erkrankungen auf [13]. Ein Einzelfall? Nein! Bei 17 von 36 analysierten Cluster-Randomisierungsstudien war nicht auszuschliessen, dass ein Selektionsbias auf individueller Ebene zum Studienergebnis beigetragen hat [14].

\section{Ethische Probleme}

Legen wir zu viel Gewicht auf individuelle Rechte und behindern damit den medizinischen Fortschritt? Meine grundsätzliche Meinung hierzu habe ich bereits vor kurzem in einem Leserbrief an die SÄZ zum Ausdruck gebracht [15], jetzt möchte ich sie etwas differenzierter darstellen. Die ethische Problematik des Zelen-Designs und von Cluster-Randomisierungsstudien besteht darin, dass den Versuchspersonen wesentliche Informationen über das Studienziel, die Randomisierung und die Therapiealternative vorenthalten werden können. Stattdessen werden sie im Glauben gelassen, die für sie ausgewählte, individuell optimale Behandlung zu erhalten [16]. Die Problematik verschärft sich dadurch, dass die Versuchspersonen den untersuchten Massnahmen nicht neutral gegenüberstehen, sondern eine eindeutige Präferenz haben (von der damit verbundenen Kontaminationsgefahr leiten sich die Studiendesigns mit unvollständiger Aufklärung ja ab): Den Kontrollpersonen wird damit die für sie attraktivere Alternative vorenthalten. In einer Studie mit Zelens Double Consent Design haben die Versuchspersonen zudem nur die Wahl, die angebotene Behandlung anzunehmen oder abzulehnen, nicht jedoch, sich der Studie als solcher zu entziehen [7], was im Heilmittelbereich im deutlichen Widerspruch zum Recht steht, die Einwilligung zur Studienteilnahme jederzeit zurückziehen zu können $[17,18]$.

Immer wieder einmal wird der «Doppelstandard» in die Diskussion eingebracht, nämlich dass im Gegensatz zur ärztlichen Routine die Gabe der Standardtherapie im Rahmen einer Studie einen komplexen Aufklärungsprozess erfordert [19]. Es besteht jedoch ein Unterschied, ob eine Behandlung in rein therapeutischer Absicht erfolgt oder Bestandteil einer Studie ist - oder anders ausgedrückt: ob sie im Kontext eines Verhältnisses von Arzt und Patient oder von Forscher und Versuchsperson steht. Im Alltag kann der Arzt die Dosierung oder die Therapie jederzeit ändern, 
während diese Optionen im Rahmen einer kontrollierten Studie eingeschränkt oder unmöglich sind. Hinzu kommt, dass praktisch jede Studie zusätzliche Arztbesuche und Untersuchungen erfordert.

Am wichtigsten ist aus meiner Sicht, mit einer Versuchsperson transparent, ehrlich und so mit dem nötigen Respekt umzugehen. Dazu gehört vor Einschluss in eine Studie die vollständige Aufklärung über ihren Zweck, ihren Ablauf sowie alle mit ihr verbundenen Vor- und Nachteile - d.h. Aufwand, Belastung und Risiko, und seien sie noch so gering. Entsprechend ist heute bei Heilmittelstudien die vorgängige und vollständige Aufklärung einer Versuchsperson über alle wesentlichen Aspekte der Studie nicht nur Standard, sondern für den Forscher verpflichtend $[17,18]$. Selbst medizinische Interventionen mit minimalem Risiko können unerwünschte Wirkungen zeigen, und kein Heilmittel ist frei davon. Das Verschweigen der Randomisierung oder einer Placebo-Kontrolle käme einer bewussten Täuschung gleich. Auch die Zustimmung zum Vorenthalten von Informationen [20] ist, abgesehen davon, dass sie das Vertrauen in Forscher und Forschung nicht unbedingt stärkt, mit dem Charakter einer informierten Einwilligung nicht vereinbar denn eine Abschätzung aller Nachteile und Risiken kann die Versuchsperson nicht vornehmen.

\section{Zelen verfolgte ursprünglich die Absicht, mit dem Verschweigen der Randomisierung die Bereitschaft zur}

\section{Studienteilnahme zu steigern}

\section{Schlussfolgerungen}

- Es gibt keine validen Daten dazu, wie gross das Problem einer Kontamination tatsächlich ist. Es ist daher auch nicht klar, ob eine vollständige Aufklärung der Versuchspersonen einen bedeutenden Kontaminationsbias erzeugt. Auf der anderen Seite sind Studiendesigns mit unvollständiger Aufklärung mit erheblichen methodologischen und ethischen Defiziten verbunden. Selbst im Fall, dass eine Kontamination ein Problem darstellt, könnte die Durchführung einer herkömmlichen randomisierten Studie mit vollständiger Aufklärung immer noch die beste Alternative sein [10]. «Zwingende methodische Gründe» zu finden, um eine Studie mit unvollständiger Aufklärung durchzuführen, wird daher schwierig sein.

- Diese Gründe können im Public Health-Bereich in Ausnahmefällen vorliegen, vorausgesetzt, dass der Fragestellung eine elementare Bedeutung zukommt und die Kontaminationsgefahr real ist [10]. Eine unvollständige oder verzögerte Aufklärung ist dagegen nicht gerechtfertigt, den Aufklä- rungs- und Rekrutierungsprozess zu erleichtern und Forschern die Arbeit abzunehmen. Interessant ist, dass Zelen ursprünglich genau diese Absicht verfolgte, nämlich mit dem Verschweigen der Randomisierung die Bereitschaft zur Studienteilnahme zu steigern [21]. Bei mehr als einem Fünftel der publizierten Studien mit Zelen-Design waren in der Tat absehbare Schwierigkeiten mit der Patientenrekrutierung der Grund zur Wahl des Studiendesigns [6] - diese Studien hätten meines Erachtens kein positives Votum von den zuständigen Ethikkommissionen erhalten dürfen. Ähnlich ist ein allgemein gehaltener Consent zu beurteilen, denn ausser der Erleichterung des Aufklärungsprozesses sind keine Vorteile ersichtlich - zwingende methodische Gründe liegen schon gar nicht vor.

- Ethikkommissionen kommt zur Beurteilung von Studien mit modifizierter Aufklärung eine tragende Rolle zu. Der Aufklärungsprozess verdient besondere Beachtung. Gibt es Unterschiede bei der Aufklärung von Patienten, je nachdem ob sie in der Interventions- oder Kontrollgruppe sind? Wird die Randomisierung der Massnahmen unnötig verschwiegen? Einen hohen Stellenwert hat auch die Bewertung von "minimalen Risiken». Dass hierzu klare Definitionen notwendig sind, zeigt zum Beispiel eine Umfrage unter Präsidenten von amerikanischen Institutional Review Boards (diese entsprechen unseren Ethikkommissionen) die Einschätzung des durch verschiedene diagnostische Verfahren induzierten Risikos unterliegt einer sehr grossen Spannbreite [22]. Ist ein minimales Risiko ein akzeptables Risiko im Rahmen einer positiven Nutzen-Risiko-Abwägung oder darf es über das Risiko des normalen Alltagslebens nicht hinausgehen, entsprechend der US-amerikanischen Regelung [23]?

- Studientypen, die für die Forschung im Public Health-Bereich in Frage kommen können, spielen für reine Heilmittelstudien praktisch keine Rolle; im Gegenteil, Heilmittelstudien mit unvollständiger Aufklärung sind im globalen regulatorischen Kontext und gemäss der aktuellen Schweizerischen Heilmittelgesetzgebung sowohl aus ethischen als auch methodologischen Gründen nicht vorstellbar. In aller Regel können diese Studien ausreichend verblindet werden, zumindest auf Patientenebene, und der möglicherweise vorliegenden Gefahr einer Kontamination stehen auf der anderen Seite die vielfältigen Formen eines Bias durch alternative Studiendesigns gegenüber. Bei Heilmittelstudien besteht daher kein Bedarf für eine Ausnahmeregelung.

- Der Gesetzgeber wird aufgefordert, spätestens auf Verordnungsstufe die nötigen Präzisierungen des Humanforschungsgesetzes vorzunehmen. 


\section{Literatur}

1 Entwurf eines Bundesgesetzes über die Forschung am Menschen (21. Oktober 2009). www.bag.admin.ch/ themen/medizin/00701/00702/07558/index. html?lang=de

2 Fässler M, Biller-Andorno N. Informed Consent Balance zwischen Patientenschutz und Forschungsinteressen. Schweiz Ärztezeitung. 2010;91(12): 499-503.

3 Zelen M. Randomized consent designs for clinical trials: an update. Stat Med. 1990;9:645-56.

4 Zelen M. Strategy and alternate randomized designs in cancer clinical trials. Cancer Treat Rep. 1982;66:1095-100.

5 Anderson J, Cockayne S, Puffer S, Torgerson DJ. Review of randomized trials using the post-randomised consent (Zelen's) design. Contemp Clin Trials. 2006;27:305-19.

6 Schillings R, Kessels AG, ter Riet G et al. Randomized consent designs in randomized controlled trials: systematic literature search. Contemp Clin Trials. 2006;27:320-32.

7 Ellenberg SS. Special Report. Randomization designs in comparative clinical trials. N Engl J Med 1984;310:1404-8.

8 Altman DG, Whitehead J, Parmar MKB et al. Randomised consent designs in cancer clinical trials. Eur J Cancer. 1995;31A:1934-44.

9 Mansour EG, Gray R, Shatila AH et al. Efficacy of adjuvant chemotherapy in high-risk node-negative breast cancer. N Engl J Med. 1989;320:485-90.

10 Torgerson DJ. Contamination in trials: is cluster randomization the answer? BMJ. 2001;322:355-7.

11 Alexander FE, Anderson TJ, Brown HK et al. 14 years of follow-up from the Edinburgh randomized trial of breast-cancer screening. Lancet. 1999;353:1903-8.
12 Giraudeau B, Ravaud P. Preventing bias in cluster randomized trials. PLoS Medicine. 2009;6:e1000065. doi:10.1371/journal.pmed.1000065

13 Streptoe A, Doherty S, Rink E et al. Behavioural counseling in general practice for the promotion of healthy behavior among adults at increased risk of coronary heart disease: randomized trial. BMJ. 1999;319:943-8.

14 Puffer S, Torgerson DJ, Watson J. Evidence for risk of bias in cluster randomized trials: review of recent trials published in three general medical journals. BMJ. 2003;327:785-9.

15 Kleist P. Balance oder fauler Kompromiss? Schweiz Ärztezeitung. 2010;91(16):624.

16 Ellenberg SS. Informed consent: protection or obstacle? Some emerging issues. Control Clin Trials. 1997;18:628-36.

17 Verordnung über klinische Versuche mit Heilmitteln (VKlin) vom 17. Oktober 2001, Art. 4 und 6. SR 812.214. www.admin.ch/ch/d/sr/8/812.214.2.de.pdf

18 International Conference on Harmonisation (ICH). E6 Guideline for Good Clinical Practice. June 1996. www.ich.org/LOB/media/MEDIA482.pdf

19 Truog RD, Robinson W, Randolph A, Morris A. Is informed consent always necessary for randomized, controlled trials? N Engl J Med. 1999;340:804-7.

20 Boter H, van Delden JJM, de Haan RJ et al. Modified informed consent procedure: consent to postponed information. BMJ. 2003;327:284-5.

Zelen M. A new design for randomized clinical trials. N Engl J Med. 1979;300:1242-5.

22 Shah S, Whittle A, Wilfond B et al. How do IRBs apply the federal risk and benefit standards for pediatric research? JAMA. 2004;291:476-82.

23 US Federal Register. Protection of Human Subjects. 1991, 45 CFR 46. 